\title{
Effect of cataract surgery on subfoveal choroidal and ganglion cell complex thicknesses measured by enhanced depth imaging optical coherence tomography
}

\author{
This article was published in the following Dove Press journal: \\ Clinical Ophthalmology \\ I November 2016 \\ Number of times this article has been viewed
}

\section{Erkan Celik \\ Burcin Cakır \\ Elif Betul Turkoglu \\ Emine Doğan \\ Gursoy Alagoz}

Sakarya University Medical Education and Research Hospital, Sakarya, Turkey
Correspondence: Erkan Celik

Sakarya University Medical Education

and Research Hospital, Sakarya 54000,

Turkey

Tel +905326605657

Email drerkancelik@gmail.com
Purpose: We aimed to evaluate the effect of cataract surgery on subfoveal choroidal thickness (CT) and ganglion cell complex (GCC) thickness, as measured by enhanced depth imagingoptical coherence tomography (OCT).

Methods: This prospective study included 30 eyes of 30 patients who had undergone uneventful phacoemulsification surgery for senile cataract but had no previous ocular surgery or other ocular abnormality. Best-corrected visual acuity, slit-lamp biomicroscopy, intraocular pressure, axial length, and central corneal thickness were measured preoperatively. The operative times (OTs) and effective phaco times were also recorded in each case. OCT measurements were performed at the preoperative visit and 1 month after cataract surgery. Study of CT and GCC thickness changes was the primary objective, but central macular thickness (CMT) and peripapillary retinal nerve fiber layer (RNFL) thicknesses were also obtained by OCT.

Results: The mean subfoveal CT was $294.4 \pm 39.2 \mu \mathrm{m}$ preoperatively and $301.4 \pm 39.9 \mu \mathrm{m}$ postoperatively $(P<0.001)$. The mean GCC thickness was $85.0 \pm 4.4 \mu \mathrm{m}$ preoperatively and $89.2 \pm 5.3 \mu \mathrm{m}$ postoperatively $(P<0.001)$. The mean CMT was $247.9 \pm 17.6 \mu \mathrm{m}$ preoperatively and $249.0 \pm 17.8 \mu \mathrm{m}$ postoperatively $(P=0.029)$. The mean RNFL thickness was $97.4 \pm 5.4 \mu \mathrm{m}$ preoperatively and $101.7 \pm 5.6 \mu \mathrm{m}$ postoperatively $(P<0.001)$. Regression analysis showed that age, sex, axial length, central corneal thickness, operative time, and effective phaco time were not associated with $\mathrm{CT}$ changes $(P=0.834, P=0.129, P=0.203, P=0.343, P=0.547$, and $P=0.147$, respectively) and GCC thickness changes ( $P=0.645, P=0.542, P=0.152, P=0.664, P=0.448$, and $P=0.268$, respectively) after cataract surgery.

Conclusion: Our results indicate that all subfoveal CT, CMT, as well as RNFL and GCC thicknesses are slightly affected after uneventful phacoemulsification surgery. After cataract surgery, the examiners should consider obtaining new baseline measurements.

Keywords: cataract, choroidal thickness, ganglion cell complex

\section{Introduction}

Optical coherence tomography (OCT) is a noninvasive method that evaluates biological tissues by in vivo imaging. ${ }^{1}$ Since its introduction, OCT has undergone several improvements and revolutionized the diagnostic, monitoring, and therapeutic approaches to many retinal diseases and glaucoma. Computerized algorithms can be used on the high-resolution images obtained by modern OCT devices in order to identify and measure the thicknesses of discrete retinal layers, including the retinal nerve fiber layer (RNFL), macular ganglion cell complex (GCC), and choroid. ${ }^{2,3}$ 
The choroid is the most vascular tissue in the eye and is known to have an important role in ocular nutrition, volume regulation, and temperature control. ${ }^{4}$ Choroidal abnormalities are critical to the onset and progression of many chorioretinal disorders such as age-related macular degeneration (AMD), polypoidal choroidal vasculopathy, Vogt-Kayanagi-Harada disease, and central serous chorioretinopathy. ${ }^{5}$ With the recent development of enhanced depth imaging (EDI), in vivo assessment of the choroid has become an area of interest. EDI allows better visualization and quantitative evaluation of the choroid, which was not possible before. Information about the choroidal thickness (CT) is useful in many clinical situations for decision making regarding the management and monitoring of disease progression. ${ }^{6}$

Neurodegenerative diseases such as glaucoma preferentially affect the GCC, which is the sum of the three innermost layers: the RNFL, the retinal ganglion cell layer, and the inner plexiform layer. These contain the axons, cell bodies, and the dendrites of the retinal ganglion cells, respectively.,8 Recent studies have shown that measurement of macular GCC thickness has the same diagnostic potential for glaucoma as RNFL thickness. ${ }^{9-11}$ Moreover, it has been reported that measurements of average GCC thickness had better sensitivity in detecting changes in retinal thickness in multiple sclerosis neurodegeneration than RNFL thickness measured by OCT. ${ }^{12}$

Both CT and GCC thickness measurements can be affected by several factors such as pupil size, scan quality, and cataract. In this study, we aimed to evaluate the effect of uneventful cataract surgery on subfoveal CT and GCC thickness measured by EDI-OCT.

\section{Patients and methods \\ Patients}

This prospective study included consecutive patients who had undergone uneventful phacoemulsification surgery for senile cataract at Sakarya University Medical Education and Research Hospital between November 2013 and June 2015. The present study was approved by the Institutional Review Board of Sakarya University Medical Education and Research Hospital and adhered to the tenets of the Declaration of Helsinki and all patients signed informed consent after they received an explanation of the nature and possible consequences of the procedure.

Exclusion criteria included a history of prior ocular surgery and systemic/ocular diseases such as diabetes mellitus, hypertension, glaucoma, AMD, diabetic retinopathy, retinal vein occlusion, uveitis, or other inflammatory and vascular pathologies. Patients with unstable fixation or dense cataracts leading to a poor scan quality of OCT images and eyes with increase in postoperative intraocular pressure (IOP) or cystoid macular edema were also excluded.

All patients had a full ophthalmic examination, including best-corrected visual acuity (BCVA) assessment, as well as slit-lamp biomicroscopy and dilated fundoscopy evaluation, and IOP measurement by Goldmann applanation tonometry. Axial length (AL) was measured by immersion echography (Axis II; Quantel Medical Inc., Cedex, France) and central corneal thickness (CCT) was measured by ultrasonographic pachymetry (Pacline; Optikon 2000 SpA, Rome, Italy). OCT measurements were performed at the preoperative visit and 1 month after cataract surgery.

\section{Cataract surgery}

Prior to the operation, tropicamide $1 \%$ and phenylephrine hydrochloride $2.5 \%$ were applied to patients' eyes for pupil dilation. Operations were performed under topical anesthesia achieved using proparacaine hydrochloride $0.5 \%$. Eyelids, eyelashes, and conjunctiva were disinfected with povidoneiodine $5 \%$. BSS PLUS ${ }^{\circledR}$ (Alcon Laboratories, Inc., Fort Worth, TX, USA) was used as infusion fluid. Phacoemulsification was performed by one experienced surgeon (EC) using INFINITI $^{\circledR}$ Vision System (Alcon Laboratories, Inc.). A clear corneal incision of $2.8 \mathrm{~mm}, 600 \mathrm{mmHg}$ vacuum, an aspiration flow rate of $30 \mathrm{~mL} / \mathrm{min}$, bottle height of $75 \mathrm{~cm}$, and quickchop technique were used in all surgeries. Two different dispersive viscoelastics (Viscoat ${ }^{\circledR}$ and BiVISC ${ }^{\text {TM}}$ ) and two different cohesive viscoelastics (Healon ${ }^{\circledR}$ and Bio-Hyalur) were used during cataract surgery. One-piece hydrophobic acrylic intraocular lens (Sensar ${ }^{\circledR}$ model: AAB00; Abbott Medical Optics Inc., Santa Ana, CA, USA) was used in all surgeries. Viscoelastics were cleaned out with marked attention, even behind the intraocular lenses. The procedure ended with intracameral cefuroxime axetil $1 \mathrm{mg} / 0.1 \mathrm{~mL}$ for prophylaxis for endophthalmitis. The operative times (OTs) and effective phaco times (EPTs) were recorded in each case. No intraoperative complications occurred. Postoperatively, all patients were treated with topical antibiotics (ofloxacin $0.3 \%$ ) for 1 week and topical steroid drops (prednisolone acetate 1\%) for 4 weeks.

\section{OCT measurements}

Cirrus EDI-OCT (Carl Zeiss Meditec AG, Jena, Germany; software version 6.5.0.772) was used to obtain subfoveal $\mathrm{CT}$ and GCC thickness measurements using high-definition one-line raster and ganglion cell analysis (macular cube $512 \times 128$ ) scan protocols, respectively. Scans with signal strength $\geq 6$ were used for analysis. Scans with misalignment, segmentation failure, decentration of the measurement circle, and poor illumination, or those out of focus, were also excluded. The one-line 
raster is a $6 \mathrm{~mm}$ line consisting of 4,096 A-scans. Scan 3 of the five scans taken, which passes through the fovea, was used for all measurements. CT was defined as the vertical distance from the outer portion of the hyperreflective line corresponding to Bruch's membrane beneath the retinal pigment epithelium to the outermost hyperreflective line of the inner scleral border. To avoid the effects of mydriatic agents or diurnal changes, all measurements were obtained under nonmydriatic conditions between $3 \mathrm{pm}$ and $5 \mathrm{pm}$. Two observers determined the measurements. Each observer measured the same eye twice. The CT was measured from the subfoveal region by using the Cirrus linear measurement tool. When there was a discrepancy of $>10 \%$ of the mean of the two values between the observers in two eyes in the study, observers discussed and repeated the measurements, and the average results from the two observers were determined as the final CT readings.

The Cirrus OCT device allows a quantitative assessment of the ganglion cell and inner plexiform layers (GCIPL) in six circular sectors centered in the fovea. It also gives information on the mean and minimum GCIPL thickness for each eye and compares these figures with a normative database. Macular cube $512 \times 128$ acquisition protocol generates a cube through a $6 \mathrm{~mm}$ square grid of 128 B-scans, each composed of 512 A-scans. A built-in GCIPL analysis algorithm detects and measures the thickness of the macular GCIPL within a $6 \times 6 \times 2 \mathrm{~mm}$ elliptical annulus area centered on the fovea. The annulus has an inner vertical diameter of $1 \mathrm{~mm}$, which was chosen to exclude the portions of the fovea where the layers are very thin and difficult to detect accurately, and an outer vertical diameter of $4 \mathrm{~mm}$, which was chosen according to where the ganglion cell layer again becomes thin and difficult to detect. The Ganglion Cell Analysis algorithm identifies the outer boundaries of the RNFL and the inner plexiform layer. The difference between the RNFL and the inner plexiform layer outer boundary segmentations yields the combined thickness of the retinal GCIPL. Similar to other Cirrus printouts, it provides GCIPL measurements in six wedge-shaped sectors with a pseudocolor scheme. The GCIPL thicknesses in the normal range are represented by green backgrounds. Those that are abnormal at the $<5 \%$ and at the $<1 \%$ level are represented by yellow and red backgrounds, respectively.

The primary objective of this study was to evaluate the subfoveal CT and average GCC thickness changes; however, central macular thickness (CMT) and peripapillary RNFL thickness were also obtained by the macular cube $512 \times 128$ and optic disc cube $200 \times 200$ protocols, respectively, using the Cirrus OCT device.

Statistical analysis was performed using SPSS Statistics for Windows, version 20.0 (IBM Corporation 2011, Armonk,
NY, USA). Paired-samples $t$-test was used for equality of means, and analysis of variance regression and Pearson correlation analysis were used for correlations between parameters.

\section{Results}

A total of 30 eyes of 30 patients (16 male and 14 female), with a mean age of $60.2 \pm 4.2$ years were included in this study. Sixteen right eyes and 14 left eyes were examined. Mean BCVA improved in all eyes, and this improvement was statistically significant $(0.46 \pm 0.15$ logarithm of minimum angle of resolution [logMAR] units preoperatively and 0.06 $\pm 0.49 \log$ MAR units postoperatively; $P<0.001$ ). Mean preoperative AL was $22.8 \pm 0.6 \mathrm{~mm}$ (range: $21.5-24.1 \mathrm{~mm}$ ) and mean CCT was $551.5 \pm 22.1 \mu \mathrm{m}$. Mean OT was $8.6 \pm 2.7$ minutes and mean EPT was 6.9 \pm 2.5 seconds (Table 1).

The mean subfoveal CT was 294.4 $\pm 39.2 \mu \mathrm{m}$ (range: 201-356 $\mu \mathrm{m}$ ) preoperatively and 301.4 $\pm 39.9 \mu \mathrm{m}$ (range: 231-367 $\mu \mathrm{m})$ postoperatively. The difference was statistically significant $(P<0.001)$. The mean GCC thickness was $85.0 \pm 4.4 \mu \mathrm{m}$ (range: $78-94 \mu \mathrm{m}$ ) preoperatively and $89.2 \pm 5.3 \mu \mathrm{m}$ (range: $81-99 \mu \mathrm{m}$ ) postoperatively. The difference was statistically significant $(P<0.001)$ (Figure 1$)$. The mean CMT was $247.9 \pm 17.6 \mu \mathrm{m}$ preoperatively (range:

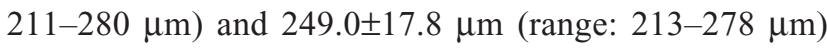
postoperatively. The difference was statistically significant $(P=0.029)$. The mean RNFL thickness was 97.4 $\pm 5.4 \mu \mathrm{m}$ preoperatively (range: $87-110 \mu \mathrm{m}$ ) and $101.7 \pm 5.6 \mu \mathrm{m}$ (range: 91-113 $\mu \mathrm{m})$ postoperatively. The difference was statistically significant $(P<0.001)$ (Table 2$)$.

Regression analysis showed that age, sex, AL, CCT, $\mathrm{OT}$, and EPT were not associated with $\mathrm{CT}$ changes ( $P=0.834, P=0.129, P=0.203, P=0.343, P=0.547$, and $P=0.147$, respectively) and GCC thickness changes $(P=0.645, P=0.542, P=0.152, P=0.664, P=0.448, P=0.268$, respectively) after cataract surgery.

Table I Clinical characteristics of patients

\begin{tabular}{ll}
\hline Clinical characteristics of patients & Results \\
\hline Mean age, years & $60.2 \pm 4.2$ \\
Male/female, $\mathrm{n}$ & $16 / 14$ \\
Right/left eye, $\mathrm{n}$ & $16 / 14$ \\
Preoperative/postoperative BCVA, logMAR units & $0.46 \pm 0.15 / 0.06 \pm 0.49$ \\
Mean AL, mm & $22.8 \pm 0.6$ \\
Mean CCT, $\mu \mathrm{m}$ & $551.5 \pm 22.1$ \\
Mean OT, minutes & $8.6 \pm 2.7$ \\
Mean EPT, seconds & $6.9 \pm 2.5$
\end{tabular}

Note: Data presented as mean \pm standard deviation.

Abbreviations: AL, axial length; BCVA, best-corrected visual acuity; $C C T$, central corneal thickness; EPT, effective phaco time; logMAR, logarithm of minimum angle of resolution; OT, operative time. 

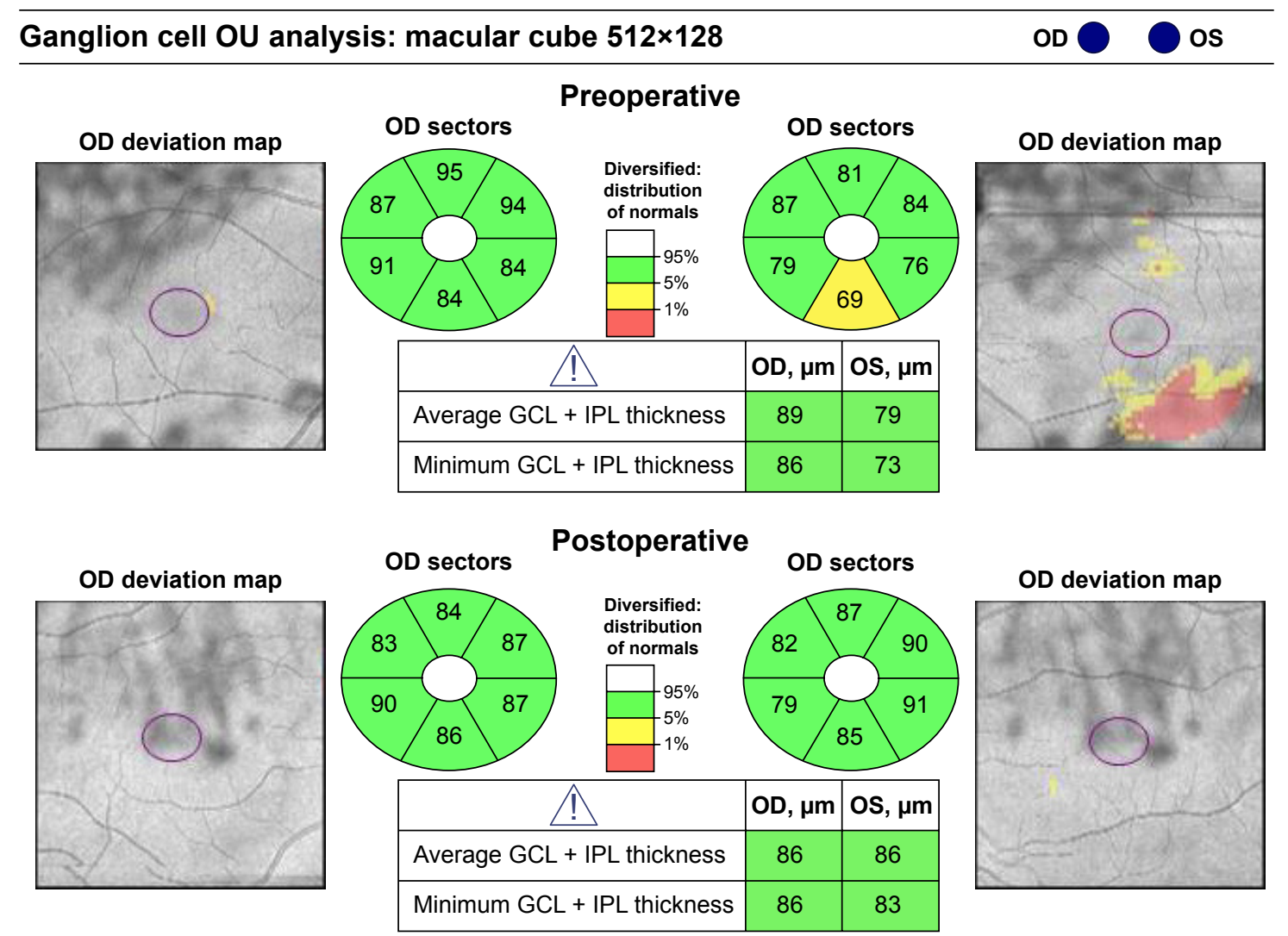

Figure I GCC thickness analysis of a patient who underwent uneventful left cataract surgery.

Notes: Note that preoperative average GCC thickness was $79 \mu \mathrm{m}$ and, postoperatively, it was $86 \mu \mathrm{m}$. Circles indicate fovea.

Abbreviations: GCC, ganglion cell complex; GCL, ganglion cell layer; IPL, inner plexiform layer; OD, oculus dexter (the right eye); OS, oculus sinister (the left eye); OU, oculus uterque (both eyes).

\section{Discussion}

In this study, we investigated the effect of cataract surgery on subfoveal CT and GCC thickness, as measured by EDI-OCT, and we found that both subfoveal CT and GCC thickness increased slightly after cataract surgery. CMT and peripapillary RNFL thicknesses were also evaluated as a secondary objective, and we observed statistically significant increases in CMT and RNFL thickness. Our results indicate that CMT, subfoveal CT, as well as RNFL and GCC thicknesses are slightly affected after phacoemulsification surgery. Although our study included a small group (only 30 eyes), we assume that our

Table 2 Preoperative and postoperative OCT parameters

\begin{tabular}{llll}
\hline OCT parameters & Preoperative & Postoperative & $P$-value \\
\hline Subfoveal CT, $\mu \mathrm{m}$ & $294.4 \pm 39.2$ & $301.4 \pm 39.9$ & $<0.00 \mathrm{I}$ \\
GCC thickness, $\mu \mathrm{m}$ & $85.0 \pm 4.4$ & $89.2 \pm 5.3$ & $<0.00 \mathrm{I}$ \\
CMT, $\mu \mathrm{m}$ & $247.9 \pm 17.6$ & $249.0 \pm 17.8$ & 0.029 \\
RNFL thickness, $\mu \mathrm{m}$ & $97.4 \pm 5.4$ & $101.7 \pm 5.6$ & $<0.00 \mathrm{I}$ \\
\hline
\end{tabular}

Note: Data presented as mean \pm standard deviation.

Abbreviations: CMT, central macular thickness; CT, choroidal thickness; GCC, ganglion cell complex; OCT, optical coherence tomography; RNFL, retinal nerve fiber layer. data reliability is high because of the homogeneous nature of the patients (similarities of age, cataract type and severity, CCT, AL, OT, EPT and so on) and the careful/detailed OCT examinations performed by two observers, avoiding the effects of diurnal changes and mydriatic agents. Zhao et a ${ }^{13}$ revealed significant diurnal variations of the $\mathrm{CT}$ at the macular region in young healthy female individuals. Similarly, Han et a ${ }^{14}$ found diurnal variations in $\mathrm{CT}$ in healthy volunteers.

Cataract surgery by phacoemulsification is one of the most common intraocular surgeries that improve the quality of vision. However, it is an invasive procedure leading to an inflammatory response - mostly induced by the release of prostaglandins - in the retina and choroid and, in many patients, can lead to worsening of preexisting retinal diseases such as diabetic macular edema. ${ }^{15,16}$ Recent studies have shown subclinical increase of CMT and peripapillary RNFL thickness after uneventful cataract surgery. ${ }^{17-19}$ However, the morphologic changes that this inflammatory insult produces in the choroid have not been studied well because of the lack of detailed imaging techniques for the choroid. After the introduction of EDI, changes in CT in several pathologic 
(central serous chorioretinopathy, choroidal neovascularization, and high myopia) and physiologic (age, sex, and AL) conditions can be easily assessed. ${ }^{20-22}$ Therefore, CT is being measured increasingly often and is becoming an accepted procedure for clinical and research applications.

Falcão et al $^{23}$ evaluated the effect of uneventful phacoemulsification on the subfoveal CT and CMT, and they reported that phacoemulsification induced nonpathologic increases in CMT; however, these changes were not accompanied by significant changes in CT. They claimed that the inflammatory insult due to phacoemulsification mainly affects at the retinal level and seems to be independent of CT changes. ${ }^{23} \mathrm{By}$ contrast; our results support the recent studies by Ohsugi et $\mathrm{al}^{24}$ and Noda et $\mathrm{al},{ }^{25}$ which report significant increases in CT after uneventful phacoemulsification. Ohsugi et $\mathrm{al}^{24}$ evaluated 100 eyes and emphasized that the AL and changes in IOP were critical for evaluating the changes in $\mathrm{CT}$ after cataract surgery. Noda et $\mathrm{al}^{25}$ evaluated 29 eyes and observed that the increase in subfoveal CT did not subside to baseline even at 6 months postoperatively. They also found that male sex and thicker baseline CT predicted a larger magnitude of increase in CT after cataract surgery. ${ }^{25}$ In our study, interestingly, the parameters of age, sex, AL, baseline CT, and CCT did not influence the postoperative CT statistically in regression analysis. We also analyzed the effect of OT and EPT on postoperative CT and we did not find any relationship. This may be explained by the short/similar results in terms of OTs and EPTs, as well as the experience of the surgeon conducting a quick surgery with less anterior segment manipulation, thus reducing the inflammatory response after cataract surgery.

The mechanism for the increase in CT after phacoemulsification surgery is unclear. A speculation is that it may be related to postoperative inflammation because proinflammatory prostaglandins and cytokines are considered to explain macular edema after cataract surgery, and inflammatory disorders are also known to increase the CT. ${ }^{26,27}$ Another speculation is that cataract surgery introduces more light into the eyes, leading to greater metabolic activation in the retinal pigment epithelium, causing angiogenesis and inflammation. ${ }^{28,29}$ In the Beaver Dam and Blue Mountains eye studies, ${ }^{30-32}$ it has been suggested that cataract surgery is associated with the onset of AMD, resulting in visual impairment due to neovascularization originating from the choroid. Noda et $\mathrm{al}^{25}$ report that cataract surgery influences the CT and this influence persists for as long as 6 months postoperatively and may affect the process of AMD.

Choroidal abnormalities are central to chorioretinal disorders such as AMD, polypoidal choroidal vasculopathy, and central serous chorioretinopathy. Furthermore, it has been claimed that pathologic choroidal blood supply is an important factor in open-angle glaucoma leading to optic nerve damage. ${ }^{33,34} \mathrm{CT}$ s of glaucomatous eyes have been reported to be thinner in postmortem histologic studies. In addition, significant increases in choroidal extravascular volume have been confirmed in many primary angle-closure glaucoma eyes and abnormal $\mathrm{CT}$ has been hypothesized to be a contributing feature in these eyes. ${ }^{35,36}$ However, it is unclear whether these findings represent a risk factor or a consequence of these diseases.

The hallmark of glaucoma is the loss of the retinal ganglion cell axons, which leads to a typical optic neuropathy. Qualitative and quantitative evaluations of the optic nerve head and RNFL have been used to detect evidence of glaucomatous damage. ${ }^{9-11}$ In a recent study, ${ }^{8}$ it has been suggested that the GCC thickness may be the most relevant parameter to measure in glaucoma. Nouri-Mahdavi et $\mathrm{al}^{37}$ also reported that regional GCC measurements derived from Cirrus HD-OCT performed as well as regional RNFL outcomes for the detection of early glaucoma. Moreover, macular GCC thickness has been found to have better structure-function correlations than RNFL thickness with both visual function and central nervous system findings in multiple sclerosis neurodegeneration. ${ }^{12}$

As OCT uses near-infrared light and is based on interferometry, its image quality is influenced by opacities in the optical path. Loss of OCT image quality is caused by attenuation of the light in the OCT scanning spot on the retina. ${ }^{17,18}$ Several studies have shown that lens opacity decreased image quality and postoperative RNFL thickness measurements increased significantly. ${ }^{38-40}$ The more advanced the cataract, the less is the signal quality and the thinner are the recorded RNFL and GCC thicknesses. The coexistence of neurodegenerative diseases such as glaucoma and cataract among elderly patients is not unusual, and cataract may decrease the quality of fundus images, leading to an incorrect evaluation in the diagnosis and follow-up of these patients.

Nakatani et $\mathrm{al}^{7}$ examined the effect of cataract on the measurements of GCC and RNFL thicknesses, and they reported that all thickness parameters in GCC and RNFL increased slightly after phacoemulsification surgery. We also found that the thicknesses of RNFL and GCC increased after cataract surgery. Therefore, we decided that the presence of cataract may lead to an underestimation of the thicknesses of GCC and RNFL, and this should be taken into account when analyzing progression in glaucoma and/or neurodegenerative diseases such as multiple sclerosis. After cataract surgery, the 
examiners should consider obtaining new baseline measurements, as suggested in previous studies.

This study has many limitations such as short follow-up time, relatively small sample size, and lack of eyes with glaucoma and neurodegenerative diseases. In addition, we analyzed just mean subfoveal CT and mean thickness of GCC and RNFL, leading to lack of segmentation of these parameters. Furthermore, we manually determined the choroidal-scleral interface and measured CT with Cirrus linear measurement tool. Although we used scans with signal strength $\geq 6$ for analysis and excluded poor images, the effect of signal strength index on measurements was not investigated. Furthermore, we used postoperative topical steroids after cataract surgery; thus, the true effect of phacoemulsification on OCT parameters could not be evaluated and we could not determine whether and when the increased CT can return to baseline measurements.

\section{Conclusion}

Phacoemulsification surgery increased CT and it should be kept in mind that the small changes in CT may affect the onset of AMD, which is a serious disease that results in central visual impairment. Although the mechanism is unclear, it has been postulated that $\mathrm{CT}$ changes may also play a role in glaucoma. Furthermore, all thickness parameters of GCC and RNFL measurements increased after phacoemulsification surgery. We suggest establishment of new baseline measurements after cataract surgery for monitoring patients with glaucoma and neurodegenerative diseases. Further studies with a greater number of patients and longer duration of follow-up and conducted using new computerized OCT algorithms are needed.

\section{Disclosure}

The authors report no conflicts of interest in this work.

\section{References}

1. Wojtkowski M, Bajraszewski T, Gorczyńska I, et al. Ophthalmic imaging by spectral optical coherence tomography. Am J Ophthalmol. 2004;138(3):412-419.

2. Shin JW, Shin YU, Lee BR. Choroidal thickness and volume mapping by a six radial scan protocol on spectral-domain optical coherence tomography. Ophthalmology. 2012;119(5):1017-1023.

3. Gupta P, Sidhartha E, Girard MJ, Mari JM, Wong TY, Cheng CY. A simplified method to measure choroidal thickness using adaptive compensation in enhanced depth imaging optical coherence tomography. PLoS One. 2014;9(5):e96661.

4. Manjunath V, Taha M, Fujimoto JG, Duker JS. Choroidal thickness in normal eyes measured using Cirrus HD optical coherence tomography. Am J Ophthalmol. 2010;150(3):325-329.

5. Yeoh J, Rahman W, Chen F, et al. Choroidal imaging in inherited retinal disease using the technique of enhanced depth imaging optical coherence tomography. Graefes Arch Clin Exp Ophthalmol. 2010;248(12): 1719-1728.
6. Spaide RF, Koizumi H, Pozzoni MC. Enhanced depth imaging spectral domain optical coherence tomography. Am J Ophthalmol. 2008; 146:496-500.

7. Nakatani Y, Higashide T, Ohkubo S, Takeda H, Sugiyama K. Effect of cataract and its removal on ganglion cell complex thickness and peripapillary retinal nerve fiber layer thickness measurements by fourier-domain optical coherence tomography. J Glaucoma. 2013;22(6):447-455.

8. Mwanza JC, Oakley JD, Budenz DL, Chang RT, Knight OJ, Feuer WJ. Macular ganglion cell-inner plexiform layer: automated detection and thickness reproducibility with spectral domain-optical coherence tomography in glaucoma. Invest Ophthalmol Vis Sci. 2011;52(11): 8323-8329.

9. Kim NR, Lee ES, Seong GJ, Kim JH, An HG, Kim CY. Structurefunction relationship and diagnostic value of macular ganglion cell complex measurement using Fourier-domain OCT in glaucoma. Invest Ophthalmol Vis Sci. 2010;51(9):4646-4651.

10. Seong M, Sung KR, Choi EH, et al. Macular and peripapillary retinal nerve fiber layer measurements by spectral domain optical coherence tomography in normal-tension glaucoma. Invest Ophthalmol Vis Sci. 2010; 51(3):1446-1452.

11. Tan O, Chopra V, Lu AT, et al. Detection of macular ganglion cell loss in glaucoma by Fourier-domain optical coherence tomography. Ophthalmology. 2009;116(12):2305-2314.

12. González-López JJ, Rebolleda G, Leal M, et al. Comparative diagnostic accuracy of ganglion cell-inner plexiform and retinal nerve fiber layer thickness measures by Cirrus and Spectralis optical coherence tomography in relapsing-remitting multiple sclerosis. Biomed Res Int. 2014;2014:128517.

13. Zhao M, Yang XF, Jiao X, et al. The diurnal variation pattern of choroidal thickness in macular region of young healthy female individuals using spectral domain optical coherence tomography. Int J Ophthalmol. 2016;9(4):561-566.

14. Han YS, Lim HB, Lee SH, Kim JY. Diurnal variation in choroidal and retinal thickness of the early treatment of diabetic retinopathy study macular subfields determined using swept-source optical coherence tomography. Ophthalmologica. 2015;233(3-4):192-197.

15. Kim SJ, Flach AJ, Jampol LM. Nonsteroidal anti-inflammatory drugs in ophthalmology. Surv Ophthalmol. 2010;55(2):108-133.

16. Vukicevic M, Gin T, Al-Qureshi S. Prevalence of optical coherence tomography-diagnosed postoperative cystoid macular oedema in patients following uncomplicated phaco-emulsification cataract surgery. Clin Experiment Ophthalmol. 2012;40(3):282-287.

17. von Jagow B, Ohrloff C, Kohnen T. Macular thickness after uneventful cataract surgery determined by optical coherence tomography. Graefes Arch Clin Exp Ophthalmol. 2007;245(12):1765-1771.

18. Kok PH, van den Berg TJ, van Dijk HW, et al. The relationship between the optical density of cataract and its influence on retinal nerve fibre layer thickness measured with spectral domain optical coherence tomography. Acta Ophthalmol. 2013;91(5):418-424.

19. Dada T, Behera G, Agarwal A, Kumar S, Sihota R, Panda A. Effect of cataract surgery on retinal nerve fiber layer thickness parameters using scanning laser polarimetry (GDxVCC). Indian J Ophthalmol. 2010; 58(5):389-394.

20. Margolis R, Spaide RF. A pilot study of enhanced depth imaging optical coherence tomography of the choroid in normal eyes. Am J Ophthalmol. 2009;147(5):811-815.

21. Imamura Y, Fujiwara T, Margolis R, Spaide RF. Enhanced depth imaging optical coherence tomography of the choroid in central serous chorioretinopathy. Retina. 2009;29(10):1469-1473.

22. Fujiwara T, Imamura Y, Margolis R, Slakter JS, Spaide RF. Enhanced depth imaging optical coherence tomography of the choroid in highly myopic eyes. Am J Ophthalmol. 2009;148(3):445-450.

23. Falcão MS, Gonçalves NM, Freitas-Costa P, et al. Choroidal and macular thickness changes induced by cataract surgery. Clin Ophthalmol. 2014;8:55-60.

24. Ohsugi H, Ikuno Y, Ohara Z, et al. Changes in choroidal thickness after cataract surgery. J Cataract Refract Surg. 2014;40(2):184-191. 
25. Noda Y, Ogawa A, Toyama T, Ueta T. Long-term increase in subfoveal choroidal thickness after surgery for senile cataracts. Am JOphthalmol. 2014;158(3):455-459.

26. $\mathrm{Xu} \mathrm{H}$, Chen M, Forrester JV, Lois N. Cataract surgery induces retinal pro-inflammatory gene expression and protein secretion. Invest Ophthalmol Vis Sci. 2011;52(1):249-255.

27. Liu H, Demetriades AM, Xiao WH, Campochiaro PA, Vinores SA. Mouse model of post-surgical breakdown of the blood-retinal barrier. Curr Eye Res. 2004;28(6):421-426.

28. Arany Z, Foo SY, Ma Y, et al. HIF-independent regulation of VEGF and angiogenesis by the transcriptional coactivator PGC-1alpha. Nature. 2008;451(7181):1008-1012.

29. Ueta T, Inoue T, Yuda K, Furukawa T, Yanagi Y, Tamaki Y. Intense physiological light upregulates vascular endothelial growth factor and enhances choroidal neovascularization via peroxisome proliferatoractivated receptor g coactivator-1a in mice. Arterioscler Thromb Vasc Biol. 2012;32(6):1366-1371.

30. Klein BE, Howard KP, Lee KE, Iyengar SK, Sivakumaran TA, Klein R. The relationship of cataract and cataract extraction to age-related macular degeneration: the Beaver Dam Eye Study. Ophthalmology. 2012;119(8):1628-1633.

31. Cugati S, Mitchell P, Rochtchina E, Tan AG, Smith W, Wang JJ. Cataract surgery and the 10-year incidence of age-related maculopathy: the Blue Mountains Eye Study. Ophthalmology. 2006;113(11):2020-2025.

32. Wang JJ, Klein R, Smith W, Klein BE, Tomany S, Mitchell P. Cataract surgery and the 5-year incidence of late-stage age-related maculopathy: pooled findings from the Beaver Dam and Blue Mountains eye studies. Ophthalmology. 2003;110(10):1960-1967.

33. Maul EA, Friedman DS, Chang DS, et al. Choroidal thickness measured by spectral domain optical coherence tomography: factors affecting thickness in glaucoma patients. Ophthalmology. 2011;118(8): 1571-1579.
34. Hayreh SS, Revie IH, Edwards J. Vasogenic origin of visual field defects and optic nerve changes in glaucoma. Br J Ophthalmol. 1970;54(7): $461-472$.

35. Kubota T, Jonas JB, Naumann GO. Decreased choroidal thickness in eyes with secondary angle closure glaucoma: an aetiological factor for deep retinal changes in glaucoma? Br J Ophthalmol. 1993;77(7): 430-432.

36. Yin ZQ, Vaegan, Millar TJ, Beaumont P, Sarks S. Widespread choroidal insufficiency in primary open-angle glaucoma. J Glaucoma. 1997;6(1): 23-32.

37. Nouri-Mahdavi K, Nowroozizadeh S, Nassiri N, et al. Macular ganglion cell/inner plexiform layer measurements by spectral domain optical coherence tomography for detection of early glaucoma and comparison to retinal nerve fiber layer measurements. Am J Ophthalmol. 2013;156(6): 1297-1307.

38. Sánchez-Cano A, Pablo LE, Larrosa JM, Polo V. The effect of phacoemulsification cataract surgery on polarimetry and tomography measurements for glaucoma diagnosis. J Glaucoma. 2010;19(7):468-474.

39. Mwanza JC, Bhorade AM, Sekhon N, et al. Effect of cataract and its removal on signal strength and peripapillary retinal nerve fiber layer optical coherence tomography measurements. J Glaucoma. 2011;20(1):37-43

40. Cagini C, Fiore T, Iaccheri B, Piccinelli F, Ricci MA, Fruttini D. Macular thickness measured by optical coherence tomography in a healthy population before and after uncomplicated cataract phacoemulsification surgery. Curr Eye Res. 2009;34(12):1036-1041.
Clinical Ophthalmology

\section{Publish your work in this journal}

Clinical Ophthalmology is an international, peer-reviewed journal covering all subspecialties within ophthalmology. Key topics include: Optometry; Visual science; Pharmacology and drug therapy in eye diseases; Basic Sciences; Primary and Secondary eye care; Patient Safety and Quality of Care Improvements. This journal is indexed on

Submit your manuscript here: http://www.dovepress.com/clinical-ophthalmology-journal

\section{Dovepress}

PubMed Central and CAS, and is the official journal of The Society of Clinical Ophthalmology (SCO). The manuscript management system is completely online and includes a very quick and fair peer-review system, which is all easy to use. Visit http://www.dovepress.com/ testimonials.php to read real quotes from published authors. 